\title{
Rol del riñón en la hipertensión sal-sensible
}

\author{
LEOPOLDO ARDILES, SERGIO MEZZANO
}

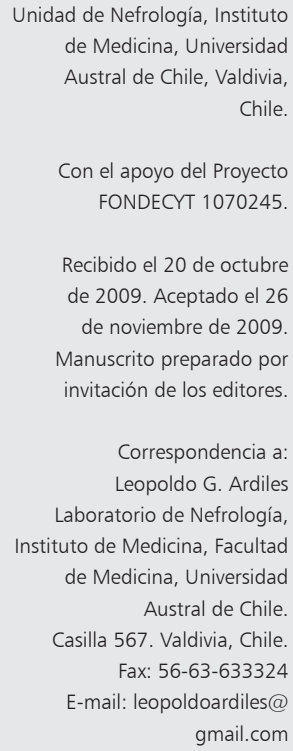

Unidad de Nefrología, Instituto de Medicina, Universidad Austral de Chile, Valdivia, Chile.

Con el apoyo del Proyecto FONDECYT 1070245.

Recibido el 20 de octubre de 2009. Aceptado el 26 de noviembre de 2009. Manuscrito preparado por invitación de los editores.

Correspondencia a: Leopoldo G. Ardiles Laboratorio de Nefrología, Instituto de Medicina, Facultad de Medicina, Universidad Austral de Chile. Casilla 567. Valdivia, Chile. Fax: 56-63-633324 E-mail: leopoldoardiles@ gmail.com

\section{Role of the kidney in salt sensitive hypertension}

An important proportion of patients with essential hypertension are salt sensitive, defined as those who experience significant blood pressure changes according to the amount of salt intake. They have a disturbance in the pressure induced natriuresis mechanism and their kidneys have functional and morphological alterations consistent with an acquired tubulointerstitial alteration, afferent arteriole damage and alteration of peritubular capillaries. All these alterations lead to disturbances in sodium load excretion under normal pressures. There is also an associated activation of kidney vasoconstrictor/salt retaining systems and a reduction in the vasodilator/ salt eliminating mechanisms. These alterations, that originate early in life, generate a new blood pressure level, that corrects natriuresis at the expense of a sustained hypertension.

(Rev Med Chile 2010; 138: 862-867).

Key words: Hypertension, renal: Kidney diseases; Natriuresis.
41 1 sodio, catión de la sal común, fue descubierto en $1807^{1} \mathrm{y}$ es encontrado normalmente en los alimentos en la forma de cloruro de sodio constituyendo un nutriente esencial, necesario para la mantención del volumen extracelular y la osmolaridad sérica ${ }^{2}$. Desde el inicio de la vida, y con la evolución de las especies, éstas fueron migrando progresivamente desde el agua a la tierra, surgiendo las aves y los mamíferos, entre ellos el hombre; éstos debieron desarrollar procesos adaptativos para desarrollarse en donde el agua y la sal eran difícilmente disponibles, destacando entre ellos la aparición del aparato yuxtaglomerular, el sistema renina-angiotensina-aldosterona e importantes cambios en la fisiología renal. La dieta humana que en sus orígenes era vegetariana, con un contenido de sal tan bajo como $0,7 \mathrm{~g} / \mathrm{día}^{3}$ cambió, con el desarrollo de la agricultura para llegar a ser, en nuestros días, francamente hipersódica. La práctica de adicionar sal, en la forma de cloruro de sodio, a la comida ha sido reconocida como un fenómeno cultural inicialmente relacionado con la preservación de los alimentos alcanzando un promedio mundial de 10 gramos diarios ${ }^{4}$, cifra que excede ampliamente las necesidades fisiológicas ${ }^{5}$ y tiene un rol patogénico no sólo en la hipertensión arterial sino también en la nefrolitiasis, asma, cáncer gástrico y osteoporosis ${ }^{1}$.

La relación entre la ingesta de sal y la presión arterial ha sido estudiada y validada en numerosos estudios epidemiológicos e intervencionales que han demostrado que el promedio de presión arterial es más alto en poblaciones cuyas dietas tienen un alto contenido de sal; por su parte, los grupos humanos que todavía se alimentan con dietas pobres en sal presentan prevalencias de hipertensión arterial casi inexistentes ${ }^{4,6,7}$. Por otra parte, la sola reducción del contenido de sal en la dieta de 10 a 5 gramos diarios por 4 semanas es capaz de reducir en $5 \mathrm{mmHg}$ la presión sistólica y en 2,7 $\mathrm{mmHg}$ la presión diastólica en individuos hipertensos ${ }^{8}$ y esta modesta variación tensional 
en relación con el cambio en la ingesta de sodio es mucho más evidente en aquellos sujetos que han sido catalogados como "sal sensibles".

Si bien la hipótesis del rol patogénico del riñón en el desarrollo de la hipertensión arterial data de los tiempos de Bright, fue sólo tras los experimentos de transmisión de HTA desde animales genéticamente hipertensos y sal-sensibles a sus controles normotensos, mediante un trasplante renal, cuando esto pudo ser afirmado con propie$\mathrm{dad}^{10}$. Estos animales, al igual que muchos sujetos hipertensos, adolecían de un defecto en la excreción de la sal, una de las funciones renales más importantes.

Inicialmente la relación riñón-hipertensión fue planteada por la observación de una reducción de la presión arterial frente a una disminución en la ingesta de sal y a ello se sumó la frecuente presencia de hipertensión arterial en la enfermedad renal crónica. Por otra parte, los sujetos normales son capaces de recibir una dieta alta en sal sin cambiar significativamente su presión arterial ${ }^{11}$. Estos hechos sugieren que la hipertensión en muchos individuos podría llevar aparejado un defecto en la excreción renal de sodio ${ }^{12}$, vinculación que tuvo su ratificación con la descripción del fenómeno de natriuresis por presión. En éste, un incremento agudo de la presión arterial es acompañado por un incremento reflejo en la excreción de sodio en tanto que ascensos tensionales más permanentes pudieran requerir de un reseteo de este mecanis$\mathrm{mo}^{13}$. La sensibilidad a la sal es un hecho frecuente entre los pacientes hipertensos describiéndose con una frecuencia global de $50 \%{ }^{14}$ aumentando con la edad ${ }^{15}$. Independiente de su efecto sobre la presión arterial, este defecto puede asociarse a hipertrofia ventricular izquierda ${ }^{16} \mathrm{y}$ sindrome metabólico ${ }^{17}$.

¿Cuáles son los mecanismos involucrados en este defecto? El riñón tiene la capacidad de producir una variedad de sustancias vasoactivas, incluyendo los sistemas renina-angiotensinaaldosterona, calicreína-cininas, prostaglandinas y óxido nítrico, pudiendo todos ellos afectar el manejo renal de sodio, el tono vascular y en consecuencia, la presión arterial ${ }^{11,18}$. Por tanto, el riñón podría generar HTA toda vez que se alteren los mecanismos involucrados en el balance de sodio ${ }^{12}$ a saber: reducción de la velocidad de filtración glomerular, aumento de la reabsorción proximal de sodio por mecanismos humorales o genéticos y la adquisición de un daño tubulointersticial.
Un primer hecho destacable es el hallazgo, tanto en pacientes hipertensos esenciales como en los modelos experimentales de sal-sensibilidad, de un aumento del grosor de la pared de la arteriola aferente ${ }^{19}$. Esta lesión vascular se asocia con una vasoconstricción cortical reduciendo el flujo plasmático renal, la velocidad de filtración glomerular y el coeficiente de ultrafiltración, teniendo como resultado directo una reducción en la filtración de sodio e indirectamente un aumento en la reabsorción tubular del mismo ${ }^{20}$.

El otro hecho importante dice relación con la presencia de un leve daño tubulointersticial adquirido. En la historia natural de la HTA esencial, es posible reconocer una fase inicial en la que el sujeto sufre alzas tensionales transitorias y episódicas, dependientes de hiperactividad del sistema nervioso y del sistema renina-angiotensina, antes de llegar a la hipertensión sostenida. Así, tanto por la acción vasoconstrictora de norepinefrina como de angiotensina II pueden gestarse la transición desde esa fase episódica o lábil a una hipertensión persistente y sal-sensible ${ }^{21}$. Estos pulsos hipertensivos iniciales pueden verse muy bien reproducidos en modelos animales inducidos por la administración sistémica de catecolaminas en los que, una vez suspendida la infusión y luego de un período de wash-out se observa la presencia de hipertensión sólo cuando los animales son sometidos a dietas hipersódicas ${ }^{22}$. Los modelos animales de sal-sensibilidad muestran característicamente un leve daño tubulointersticial evidenciado por infiltración de linfocitos y macrófagos, pérdida de la integridad de los capilares peritubulares, incremento en la expresión y actividad del sistema renina-angiotensina intrarenal, estrés oxidativo y la ya descrita remodelación de la pared de la arteriola aferente ${ }^{4}$. Este daño tubulointersticial es el producto de alteraciones en el flujo y presión capilar peritubular ${ }^{21}$, pues gran parte de la transmisión glomerular de la hipertensión sistémica que ocurre en las fases precoces o lábiles de la hipertensión esencial tiende a ser reducida gracias a la vasoconstricción de la arteriola aferente lo que resulta en su hipertrofia e isquemia anterógrada; sin embargo, este mecanismo de protección es finalmente superado transmitiéndose el estrés tensional al glomérulo y a la vasculatura postglomerular, especialmente en la región yuxtamedular donde los capilares peritubulares reclutan miofibroblastos creándose un nuevo sitio de resistencia vascular intrarenal ${ }^{23}$. 
La injuria endotelial producto de la hipertensión capilar peritubular puede afectar la producción de moléculas vasoactivas, con pérdida de actividad de la óxido nítrico sintasa (eNOS) potenciando la isquemia local ${ }^{24}$. Es así como es posible ver en las biopsias de pacientes hipertensos esenciales la reducción significativa en el número de capilares peritubulares, tal como se ve en los modelos animales ${ }^{25}$. Todo lo anterior resulta en una alteración del balance glomérulo-tubular el que funciona exageradamente debido a la activación de mediadores vasoconstrictores y la reducción de vasodilatadores en el tejido isquémico afectando el tono de la arteriola aferente y el manejo tubular del sodio $^{21}$. Recientemente se ha reconocido además un papel de las células inmunocompetentes que forman parte del infiltrado tubulintersticial y que son productoras de angiotensina II y activadoras de stress oxidativo. Estas células serían también mediadoras del estado sal-retenedor a tal punto que su reducción mediante drogas inmunosupresoras como el micofenolato de mofetil puede mejorar significativamente la sal-sensibilidad en modelos experimentales ${ }^{4}$.

Esta hipótesis fisiopatológica puede explicar la evolución natural de la hipertensión esencial con su fase inicial en la que ocurre hipertensión lábil con leve retención de sodio pero con un mecanismo de natriuresis por presión intacto; con el tiempo, las elevaciones transitorias de la presión arterial van induciendo daño capilar peritubular e inflamación por acción de vasoconstrictores e inhibición de vasodilatadores lo que altera el balance glomerulotubular (Figura 1). El engrosamiento

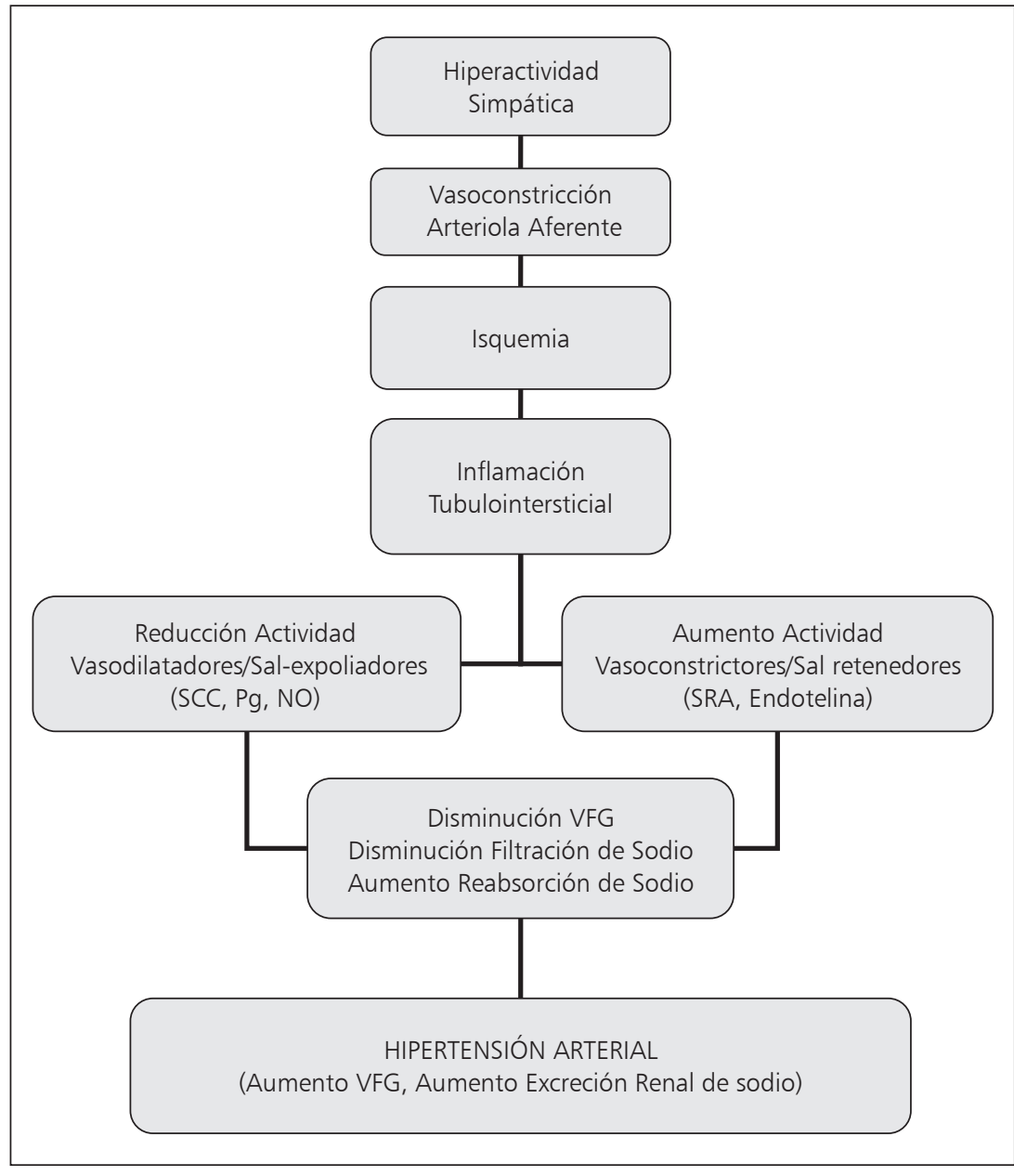

Figura 1. Mecanismos involucrados en la patogénesis de la hipertensión sensible a la sal. La alteración de la excreción de sodio eleva la presión arterial corrigiéndose la natriuresis a expensas de niveles de presión arterial más altos.

SRA = Sistema Renina-Angiotensina; $\mathrm{SCC}=$ Sistema Calicreína Cininas; $\mathrm{Pg}=$ Prostaglandinas; $\mathrm{NO}=$ Óxido nítrico; VFG: Velocidad de filtración glomerular. 


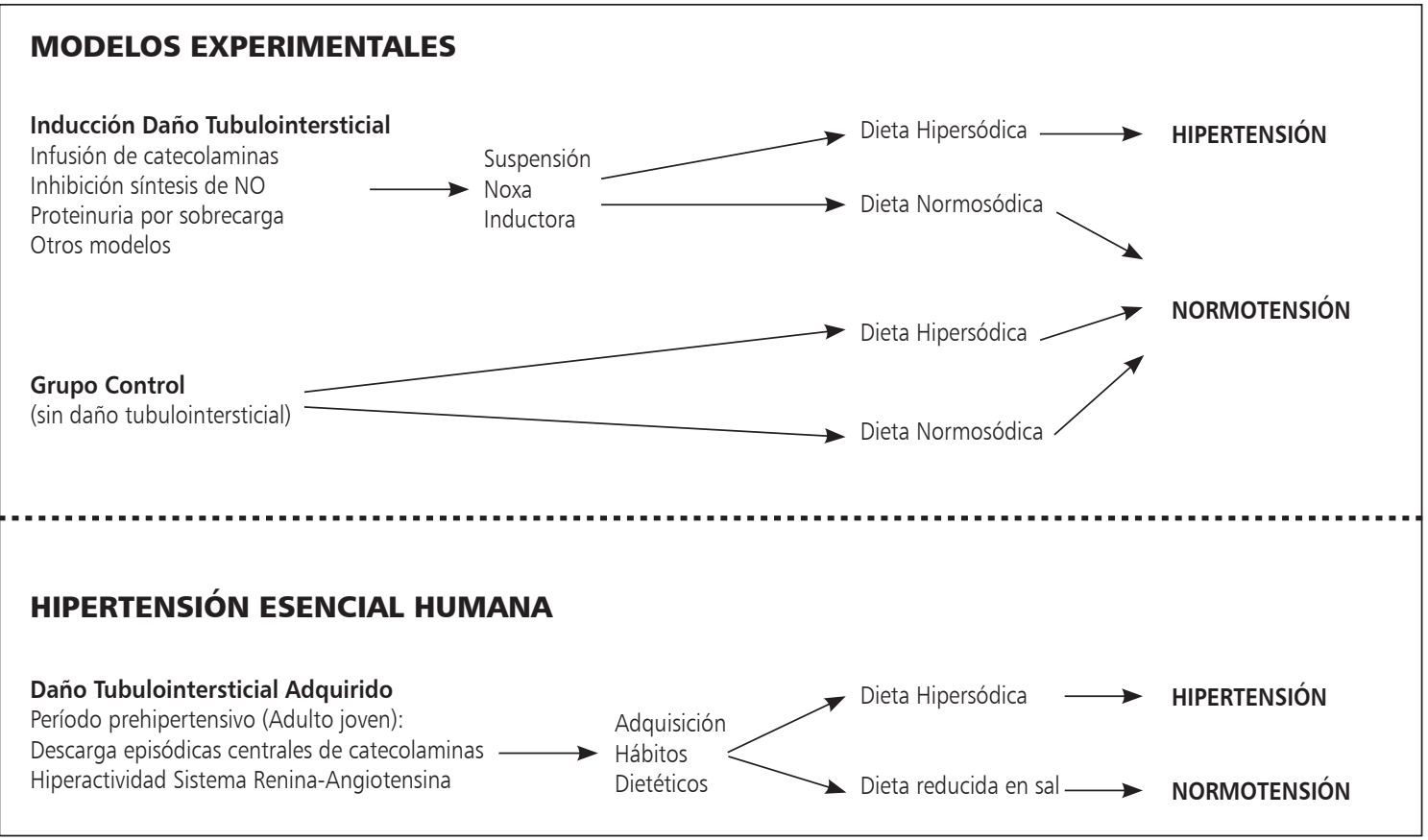

Figura 2. Hipertensión sal-sensible humana y su paralelo con los modelos experimentales. (NO: óxido nítrico)

del lecho capilar peritubular altera la gradiente y afecta la natriuresis por presión. Todo lo anterior, lleva a una reducción en la excreción renal de sodio que ante una dieta hipersódica induce expansión de volumen extracelular elevándo la presión arterial y con ello la irrigación renal, mejorando así la perfusión de las zonas isquémicas renales y restaurando el mecanismo de natriuresis, pero ahora a niveles de presión arterial más alto (Figura 1). Esta hipótesis permite explicar también porqué la salsensibilidad va aumentando en frecuencia en los grupos etarios mayores ${ }^{26}$, porqué es más frecuente en la raza negra ${ }^{27}$, en los obeso ${ }^{28}$ y porqué pacientes con artritis reumatoide o psoriasis, que además eran hipertensos, mejoran sus cifras tensionales y los marcadores urinarios de inflamación cuando son tratados con micofenolato de mofetil ${ }^{29}$.

Sin perjuicio de lo anterior, la investigación en sal-sensibilidad ha estado muy centrada en el estudio de los componentes del sistema renina angiotensina aldosterona olvidando un tanto la participación de los sistemas vasodilatadores y sal excretores. A este respecto, nuestro grupo ha dedicado su interés al estudio de la participación del sistema calicreína-cininas en la patogénesis de la sal-sensibilidad ${ }^{18}$, a través de estudios en modelos experimentales de daño renal, que dejan como secuela una hipertensión que sólo aparece al exponer a los animales a dietas ricas en sodio (Figura 2). Es así como hemos demostrado en la sobrecarga proteica exógena, un modelo animal de daño tubulointersticial no inmune, que en la fase de inducción de este daño se puede observar una severa reducción en la síntesis y excreción de calicreína ${ }^{30}$ la que se prolonga en el tiempo y se manifiesta como hipertensión sensible a la sal. Esta alteración es corregible mediante la estimulación del sistema calicreína-cininas que se obtiene mediante una dieta rica en potasio y ese efecto benéfico se pierde con el bloqueo farmacológico del receptor B2 de cininas (Ardiles et al. Stimulation of Renal Kallikrein-Kinin System Prevents Salt-Sensitive Hypertension. Resumen presentado en Renal week ASN 2005). Estos datos son coherentes con el planteamiento de otros autores que han observado sal-sensibilidad en animales con baja excreción de calicreína ${ }^{31}$ o genéticamente deficientes en cininógeno ${ }^{32}$ y aportan evidencias a la hipótesis que postula al sistema calicreína cininas renal como una válvula de escape frente a la sobrecarga de sal ${ }^{33}$ sugiriendo además un soporte fisiopatológico para el uso de sales de potasio en 
pacientes hipertensos siempre y cuando no tengan restricciones de función renal ni estén usando drogas que puedan alterar la excreción de este catión.

Hasta el momento las evidencias apoyan el concepto de que, al menos para los sujetos hipertensos, la hipertrofia ventricular izquierda es un predictor potente e independiente de eventos cardíacos, cerebrovasculares y mortalidad en general y que su reducción por un tratamiento se acompaña de protección cardiovascular. Sin embargo, esto no parece ser sólo un efecto indirecto mediado por la presión arterial sino que se ha demostrado una correlación positiva entre masa ventricular izquierda y la ingesta de sal, independiente de las cifras tensionales ${ }^{34,35}$. Adicionalmente se ha descrito una mayor prevalencia de microalbuminuria en grupos con mayor ingesta de cloruro de sodio ${ }^{36}$, mayor predisposición al daño renal crónico hipertensivo ${ }^{37}$ y alteraciones a nivel vascular como cambios en la presión de pulso y de la elasticidad vascular o a nivel celular y tisular en que una dieta alta en sodio activa mediadores pro-inflamatorios como NFkB y TNF-alfa ${ }^{38}$, incrementando la producción de superóxido ${ }^{39}$, suprimiendo la óxido nítrico sintasa ${ }^{40}$, o aumentando la actividad del sistema renina angiotensina ${ }^{41}$.

La reducción de la sal en la dieta de los hipertensos sal-sensibles puede permitir un mejor control de la presión arterial, a la vez que puede disminuir el requerimiento de medicamentos antihipertensivos, especialmente si se reemplaza por sales de potasio ${ }^{42}$. Considerando la alta ingesta de sal en la población chilena y el que una reducción moderada no induce alteraciones fisiológicas, una recomendación de este tipo es perfectamente asumible por un amplio número de personas y puede tener un efecto positivo en la reducción de las cifras de morbimortalidad cardiovascular asociadas a la hipertensión. Esto puede resultar particularmente cierto si se tiene en cuenta que con el envejecimiento de la población chilena y la epidemia de síndrome metabólico y enfermedades renales, el número de sujetos cuya presión arterial puede ser sensible a la sal es creciente.

\section{Referencias}

1. Mayer G. An update on the relationship between the kidney, salt and hypertension. Wien Med Wochenschr 2008; 158: 365-9.
2. Grollman A. The role of salt in health and disease. Am J Cardiol 1961; 8: 593-601.

3. Eaton SB, Konner M. Paleolithic nutrition. A consideration of its nature and current implications. N Engl J Med 31-1-1985; 312: 283-9.

4. Rodríguez-Iturbe B. [The role of immunocompetent cell renal infiltration in the pathogenesis of arterial hypertension]. Nefrologia 2008; 28: 483-92.

5. Franco V, Oparil S. Salt sensitivity, a determinant of blood pressure, cardiovascular disease and survival. J Am Coll Nutr 2006; 25: 247S-55S.

6. Meneton P, Jeunemaitre X, de Wardener HE, MacGregor GA. Links between dietary salt intake, renal salt handling, blood pressure, and cardiovascular diseases. Physiol Rev 2005; 85: 679-715.

7. Rodríguez-Iturbe B, Romero F, Johnson RJ. Pathophysiological mechanisms of salt-dependent hypertension. Am J Kidney Dis 2007; 50: 655-72.

8. He FJ, MacGregor GA. Effect of longer-term modest salt reduction on blood pressure. Cochrane Database Syst Rev 2004; CD004937.

9. Weinberger MH, Fineberg NS. Sodium and volume sensitivity of blood pressure. Age and pressure change over time. Hypertension 1991; 18: 67-71.

10. Dahl LK, Heine M. Primary role of renal homografts in setting chronic blood pressure levels in rats. Circ Res 1975; 36: 692-6.

11. Hayashi K. Symposium on the etiology of hypertensionsummarizing studies in 20th century. 4. Pathogenesis of hypertension-kidney as a pathogenetic organ of hypertension. Intern Med 2001; 40: 153-6.

12. Johnson RJ, Feig DI, Nakagawa T, Sánchez-Lozada LG, Rodríguez-Iturbe B. Pathogenesis of essential hypertension: historical paradigms and modern insights. J Hypertens 2008; 26: 381-91.

13. Guyton AC, Coleman TG, Cowley AV Jr, Scheel KW, Manning RD Jr, Norman RA Jr. Arterial pressure regulation. Overriding dominance of the kidneys in long-term regulation and in hypertension. Am J Med 1972; 52: 584-94.

14. Rodríguez Castellanos FE. Salt-sensitive hypertension. Arch Cardiol Mex 2006; 76 Suppl 2: S161-3.

15. Rosenthal T, Shamiss A, Holtzman E. Dietary electrolytes and hypertension in the elderly. Int Urol Nephrol 2001; 33: 575-82.

16. Morimoto A, Uzu T, Fujii T, Nishimura M, Kuroda S, Nakamura S, et al. Sodium sensitivity and cardiovascular events in patients with essential hypertension. Lancet 13-12-1997; 350: 1734-7.

17. Ritz E. Metabolic syndrome and kidney disease. Blood Purif 2008; 26: 59-62. 
18. Ardiles LG, Figueroa CD, Mezzano SA. Renal kallikreinkinin system damage and salt sensitivity: insights from experimental models. Kidney Int Suppl 2003; S2-S8.

19. Franco M, Tapia E, Santamaría J, Zafra I, García-Torres $\mathrm{R}$, Gordon KL, et al. Renal cortical vasoconstriction contributes to development of salt-sensitive hypertension after angiotensin II exposure. J Am Soc Nephrol 2001; 12: 2263-71.

20. Johnson RJ, Herrera-Acosta J, Schreiner GF, RodríguezIturbe B. Subtle acquired renal injury as a mechanism of salt-sensitive hypertension. N Engl J Med 21-3-2002; 346: 913-23.

21. Johnson RJ, Schreiner GF. Hypothesis: the role of acquired tubulointerstitial disease in the pathogenesis of saltdependent hypertension. Kidney Int 1997; 52: 1169-79.

22. Johnson RJ, Gordon KL, Suga S, Duijvestijn AM, Griffin $\mathrm{K}$, Bidani A. Renal injury and salt-sensitive hypertension after exposure to catecholamines. Hypertension 1999; 34: 151-9.

23. Johnson RJ, Alpers CE, Yoshimura A, Lombardi D, Pritzl P, Floege J, et al. Renal injury from angiotensin IImediated hypertension. Hypertension 1992; 19: 464-74.

24. Thomas SE, Anderson S, Gordon KL, Oyama TT, Shankland SJ, Johnson RJ. Tubulointerstitial disease in aging: evidence for underlying peritubular capillary damage, a potential role for renal ischemia. J Am Soc Nephrol 1998; 9: 231-42.

25. Bohle A, Muller GA, Wehrmann M, kensen-Haen S, Xiao JC. Pathogenesis of chronic renal failure in the primary glomerulopathies, renal vasculopathies, and chronic interstitial nephritides. Kidney Int Suppl 1996; 54: S2-S9.

26. Midgley JP, Matthew AG, Greenwood CM, Logan AG. Effect of reduced dietary sodium on blood pressure: a meta-analysis of randomized controlled trials. JAMA 22-5-1996; 275: 1590-7.

27. Parmer RJ, Stone RA, Cervenka JH. Renal hemodynamics in essential hypertension. Racial differences in response to changes in dietary sodium. Hypertension 1994; 24: 752-7.

28. Rocchini AP, Key J, Bondie D, Chico R, Moorehead C, Katch V, Martin M. The effect of weight loss on the sensitivity of blood pressure to sodium in obese adolescents. N Engl J Med 31-8-1989; 321: 580-5.

29. Herrera J, Ferrebuz A, MacGregor EG, Rodríguez-Iturbe B. Mycophenolate mofetil treatment improves hypertension in patients with psoriasis and rheumatoid arthritis.
J Am Soc Nephrol 2006; 17: S218-25.

30. Ardiles LG, Loyola F, Ehrenfeld P, Burgos ME, Flores CA, Valderrama G, et al. Modulation of renal kallikrein by a high potassium diet in rats with intense proteinuria. Kidney Int 2006; 69: 53-9.

31. Madeddu P, Varoni MV, Demontis MP, Pinna-Parpaglia P, Glorioso N, Anania V. Blood pressure sensitivity to salt in rats with low urinary kallikrein excretion. Immunopharmacology 1996; 33: 301-4.

32. Majima M, Yoshida O, Mihara H, Muto T, Mizogami S, Kuribayashi $\mathrm{Y}$, et al. High sensitivity to salt in kininogendeficient brown Norway Katholiek rats. Hypertension 1993; 22: 705-14.

33. Katori M, Majima M. The renal kallikrein-kinin system: its role as a safety valve for excess sodium intake, and its attenuation as a possible etiologic factor in salt-sensitive hypertension. Crit Rev Clin Lab Sci 2003; 40: 43-115.

34. Schmieder RE, Messerli FH, Garavaglia GE, Núñez BD. Dietary salt intake. A determinant of cardiac involvement in essential hypertension. Circulation 1988; 78: 951-6.

35. Swaye PS, Gifford RW Jr, Berrettoni JN. Dietary salt and essential hypertension. Am J Cardiol 1972; 29: 33-8.

36. Du CG, Ribstein J, Mimran A. Dietary sodium and target organ damage in essential hypertension. Am J Hypertens 2002; 15: 222-9.

37. Campese VM. Salt sensitivity in hypertension. Renal and cardiovascular implications. Hypertension 1994; 23: 531-50.

38. Gu JW, Tian N, Shparago M, Tan W, Bailey AP, Manning RD Jr. Renal NF-kappaB activation and TNF-alpha upregulation correlate with salt-sensitive hypertension in Dahl salt-sensitive rats. Am J Physiol Regul Integr Comp Physiol 2006; 291: R1817-24.

39. Meng S, Cason GW, Gannon AW, Racusen LC, Manning RD Jr. Oxidative stress in Dahl salt-sensitive hypertension. Hypertension 2003; 41: 1346-52.

40. Ni Z, Vaziri ND. Effect of salt loading on nitric oxide synthase expression in normotensive rats. Am J Hypertens 2001; 14: 155-63.

41. Ruan X, Wagner C, Chatziantoniou C, Kurtz A, Arendshorst WJ. Regulation of angiotensin II receptor AT1 subtypes in renal afferent arterioles during chronic changes in sodium diet. J Clin Invest 1-3-1997; 99: 1072-81.

42. Krzesinski JM, Cohen EP: Salt, the kidneys, and arterial hypertension. Acta Clin Belg 2007; 62: 348-57. 